\title{
Stroke and Stroke Mimics: Diagnosis and Treatment
}

\author{
Howard Rowley and Achala Vagal
}

\section{Learning Objectives}

- To review recent evidence based guidelines for triage in acute ischemic stroke

- To describe the imaging findings, design of CT and MRI protocols, and optimal workflow for ischemic stroke triage

- Recognize stroke mimics and offer differential considerations for emergency "Code Stroke" cases

\subsection{Introduction}

Advanced CT and MRI imaging is critical to best practice in clinical stroke care, particularly in the era of intravenous thrombolysis and intra-arterial thrombectomy treatments for appropriately selected candidates. The optimal imaging strategy will vary from site to site but needs to harness the best modality (CT versus MRI), workflow, communication, staff and provider education, expertise, and cost for the right patient at the right time. This requires a coordinated systemwide approach from regional emergency providers to inhospital care.

The over-arching goal of imaging is to establish the right diagnosis, not "just" to help direct critical triage decisions for IV thrombolysis or endovascular therapy. The following three key areas should be confidently and expeditiously evaluated in every acute stroke imaging protocol:

H. Rowley $(\varangle)$

Department of Radiology, University of Wisconsin,

Madison, WI, USA

e-mail: hrowley@uwhealth.org

A. Vagal

Department of Radiology, University of Cincinnati,

Cincinnati, $\mathrm{OH}$, USA

e-mail: vagala@ucmail.uc.edu
1. Hemorrhage: Exclude acute intracranial hemorrhage, including parenchymal hemorrhage and subarachnoid hemorrhage, as this is a critical triage branch point and exclusion for revascularization therapy.

2. Ischemia: When acute ischemic stroke signs are identified, both the extent of baseline ischemic damage and the underlying cause should be determined during the initial triage phase if possible.

3. Mimics: Stroke mimics, including seizures, tumor, infection, migraine, and other acute neurologic conditions should be detected by imaging, or at least suggested in the differential diagnosis.

\subsection{Evidence Based Guidelines for Imaging in Acute Ischemic Stroke}

There has been a recent paradigm shift in the treatment of acute ischemic stroke. Imaging is a central component in the diagnosis, triage, and selection criteria. This is reflected in the updated 2018 guidelines from American Heart Association (AHA)/ American Stroke Association (ASA) [1] and combined 2019 European Stroke Organization (ESO) and the European Society for Minimally Invasive Neurological Therapy (ESMINT) [2].

Endovascular treatment (EVT) is now established as standard of care for patients with acute ischemic stroke with large vessel occlusion (LVO) involving the anterior circulation. Large vessel occlusion is defined as occlusion involving internal carotid artery and proximal middle cerebral artery. In 2015, five randomized controlled trials (MR CLEAN, ESCAPE, REVASCAT, SWIFT-PRIME, and EXTEND-IA) [3-7] demonstrated benefit for patients with LVO within $6 \mathrm{~h}$ from symptom onset as compared to medical therapy. These patients had moderate to severe stroke deficits (NIHSS score 6) and absence of widespread established infarction on brain imaging. In 2018, two additional randomized controlled trials (DAWN and DEFUSE $3[8,9]$ ) were positive in showing benefit of EVT treatment for up to $24 \mathrm{~h}$ from the symptom onset. The late onset 
(6-24 h) trials used automated perfusion imaging including volumetric core, penumbra, and mismatch criteria for imaging selection. These trials provided level 1a evidence for benefit of advanced imaging to predict good outcomes after thrombectomy, by selecting patients with a small estimated core infarct (CBF $<30 \%$ of normal) compared to a larger symptomatic putative penumbra (clinical deficit or perfusion time prolonged $>6$ s). The DAWN trial used clinical-imaging mismatch as a selection criterion between 6 and $24 \mathrm{~h}$ from symptom onset. The DEFUSE 3 trial used mismatch and core up to $70 \mathrm{~mL}$ for patients from 6 to $16 \mathrm{~h}$ from symptom onset. An example of advanced multimodal $\mathrm{CT}$ imaging selection for thrombectomy is shown in Fig. 3.1.

Recently, two major trials have been published for extending the use of intravenous alteplase (IV tissue plasminogen activator, tPA). The WAKE-UP trial [10] used mismatch between diffusion-weighted imaging (DWI) and fluid-attenuated inversion recovery (FLAIR) on MRI and showed benefit of IV tPA versus placebo in patients with unknown time of onset and for whom mechanical thrombectomy was not performed. The EXTEND trial [11] evaluated an extended thrombolytic time window from 4.5 to $9 \mathrm{~h}$ or patients with a wake-up stroke with an uncertain onset and again showed better outcomes for IV tPA versus placebo. EXTEND used automated perfusion imaging selection (core $<70 \mathrm{~mL}$ and a mismatch $>10 \mathrm{~mL}$ ). It is expected that the guidelines will be updated soon to include the benefit of imaging selection for extended times of thrombolytic treatment (similar to the current extended EVT times).

\subsection{Protocol Strategies: Choice of Modality}

Investing in acute stroke imaging protocols yields dividends for all types of acute neurologic emergency care. With rapidly improving hardware and software, automated post-

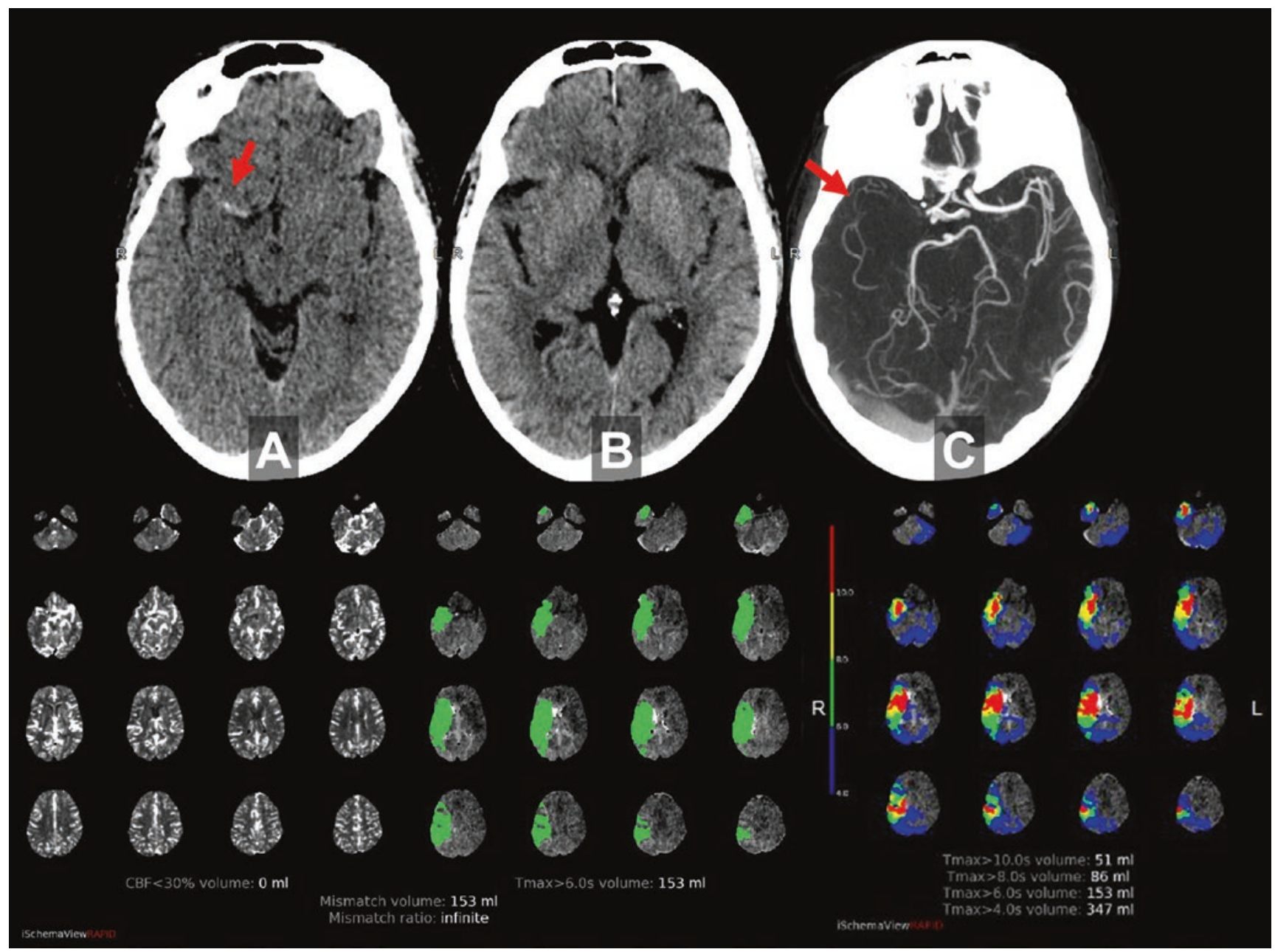

Fig. 3.1 A 75-year-old woman with acute right M1 MCA occlusion due to cardiac embolism. (a) hyperdense MCA sign (arrow); (b) normal CT $($ ASPECTS $=10)$; (c) CTA confirms right MCA occlusion with poor distal collaterals (arrow). Quantitative CT perfusion (RAPID software, lower panel) shows no estimated core infarct $(\mathrm{CBF}<30 \%)$ but a
$153 \mathrm{~mL}$ area of poor perfusion (Tmax $>6 \mathrm{~s}$ ), therefore essentially completes "target mismatch." Gradations in Tmax are shown in rainbow color. The patient underwent urgent thrombectomy with excellent recovery. (Reprinted with permission, Springer) 
processing, and artificial intelligence, rapid scanning and diagnosis increasingly facilitate emergency triage care, both in the emergency department and the inpatient setting. Either CT or MRI-based protocols work very well in acute stroke triage. However, the details are critical: these protocols require careful workflow adjustments, training, and ongoing education for rapid analysis and accurate interpretation.

Each modality has its advantages and disadvantages. $\mathrm{CT}$ is favored in most institutions world-wide due to widespread availability, inherently rapid imaging, relatively lower cost, and comfort level for referring providers and interpreting physicians; the only major potential disadvantages are radiation exposure and lack of sensitivity for brainstem/lacunar strokes and very early ischemia. MRI is arguably better, since, for example, diffusion-weighted imaging is more sensitive for detection of acute ischemia than CT, but this typically comes at higher cost, lower availability, need for MRI safety screening, and usually longer imaging exam times.

\subsection{Advanced Stroke Triage: 3C's or 4P's?}

Practical and efficient protocols have successfully been built with both $\mathrm{CT}$ and MR, ensuring accurate characterization of parenchymal injury and relevant vascular anatomy using CTA or MRA. With CT-based protocols, several groups have convincingly argued inclusion of multimodal CTA to assess for collaterals which can be particularly helpful in triage selection in the setting of large vessel occlusion. A more controversial component is perfusion imaging for either modality, particularly whether it is required for triage within $6 \mathrm{~h}$ of symptom onset. However, there is less debate for the 6- to 24-h time window, where perfusion confers valuable selection refinement based on level $1 \mathrm{~A}$ evidence from clinical trials. Mnemonics capturing these strategies, without or with perfusion, are summarized by either the 3C's: Core, Clot, and Collaterals [12]; vs the 4P's: Parenchyma, Pipes, Perfusion, and Penumbra [13]. Beyond selection for intravenous or intra-arterial therapies, perfusion imaging also enables the proper diagnosis in cases of TIA without visible parenchymal injury and can assist the reader in CTA or MRA interpretation, pointing to areas for additional scrutinywhich might have otherwise escaped detection.

\subsection{CT: Findings, Protocols, and Cases}

For most stroke centers around the world, CT is the workhorse of stroke imaging given its speed and ubiquitous availability. A CT-based stroke workup includes a non-contrast CT, CT angiogram (CTA), and CT perfusion (CTP). A non-contrast CT (NCCT) head study is the initial imaging test in a suspected case of acute ischemic stroke, most importantly to exclude hemorrhage. The early findings of acute ischemic stroke include insular hypodensity/loss of insular ribbon, hypodensity in the basal ganglia with obscuration of gray-white matter and gyral swelling. The Alberta Stroke Programme Early CT Score (ASPECTS) is a widely used objective way of assessing early ischemic changes in the middle cerebral artery distribution and uses a negative ordinal scale of 0-10 (Fig. 3.2). Low ASPECTS scores (approximately 7 or less) have been associated with increased morbidity and mortality. The recent endovascular trials used ASPECTS as a selection criterion, a score of 6 or higher suggesting a small ischemic core. Although an objective scale, ASPECTS has multiple limitations including poor interrater reliability, poor performance in setting of extensive white matter disease, and applicability only to middle cerebral arterial infarcts. Currently, there are automated ASPECTS tools using artificial intelligence (AI) that may be complimentary to human readers. The hyperdense vessel sign is another important finding on NCCT to assess intracranial thrombus. This is best visualized on thin section NCCT with MIP reconstructions which can improve sensitivity and specificity.

A CT angiogram (CTA) is now standard of care in a CT-based stroke workup to detect large vessel occlusion (LVO) and global assessment of the vascular system, including the neck. CTA has distinct advantages; it is quick, readily available, provides a comprehensive view of the arterial system, and is highly effective at identifying LVO, with an accuracy of 99\% [14]. (Ref) An LVO defined as occlusion of the ICA or MCA-M1 segment prompts consideration for EVT. An "M1 like" proximal M2 occlusion also may be considered as LVO for possible EVT, although the M2 occlusions have not been adequately studied in the EVT trials. CTA is also helpful in guiding EVT and procedural planning with depiction of extracranial vasculature for occlusions, dissections, aneurysms, or anatomic variations. Anatomic coverage of a CTA should begin at the origins of the great vessels at the aortic arch and extend through the vertex. A CTA can be performed as a single or multiphase study. A multiphasic CTA (with arterial, early venous, and late venous phases) technique can provide temporal information of collateral circulation.

An additional important piece of information that can be obtained in a CTA study is collateral circulation. Collaterals are now an established independent predictor of outcomes $[15,16]$. Good collaterals can sustain the ischemic tissue for a longer time with slower infarct growth (slow progressors), smaller final infarcts, and better outcomes. Poor collaterals are associated with faster progressors, larger infarcts, and worse outcomes in spite of successful reperfusion. Overlapping, thick section maximal intensity projection (MIP) images can be easily reconstructed on the CT scanners or on PACS stations and are helpful for LVO detection and for collateral grading (Fig. 3.1). There are numerous collateral grading scores with no consensus on optimal score; however, for practical purposes, a "good" versus "bad" dichotomized collateral information of the affected arte- 


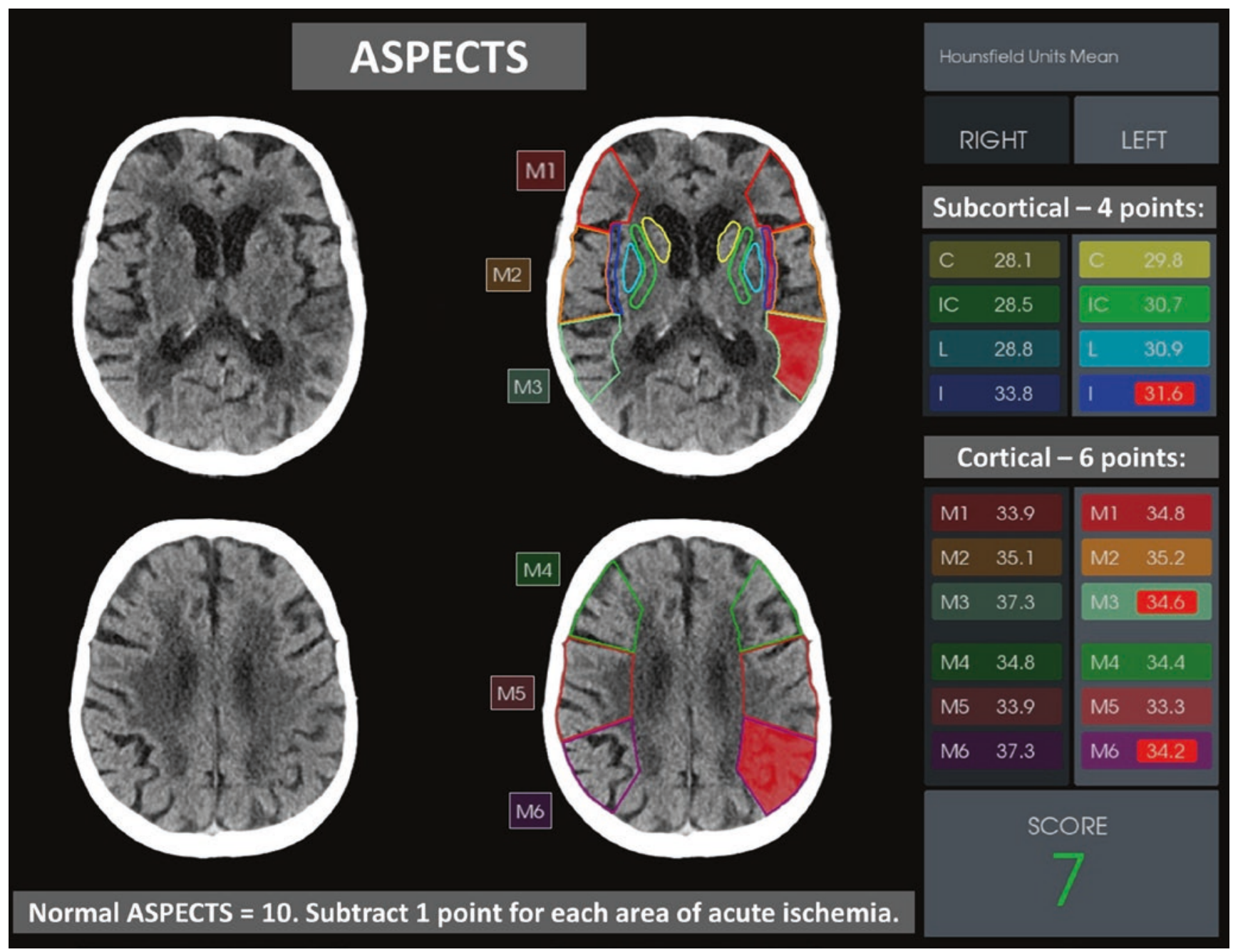

Fig. 3.2 ASPECTS (Alberta Stroke Programme Early CT Score), acute left MCA ischemia. On all $5 \mathrm{~mm}$ non-contrast axial images from basal ganglia to the centrum semiovale, four subcortical areas (caudate, internal capsule, lentiform nucleus, and insula) and six MCA sectors (M1-M6) are reviewed for signs of low attenuation suggesting acute MCA ischemia. A normal scan or one with only old lesions receives ASPECTS $=10$. A point is subtracted for each of the ten areas showing

rial territory as compared to the contralateral side is sufficient. Color coded collateral circulation displays using AI are being introduced which may aid accurate estimation of the collateral circulation, thus helping in timely patient triage.

CT Perfusion (CTP) is now increasingly used in stroke centers as part of the imaging workup, performed in conjunction with NCCT and CTA. CTP gives important information about the extent of the ischemic core (irreversibly injured tissue) and penumbra (at risk but salvageable tissue). CTP is performed in a cine mode by sequentially imaging a defined section of tissue after a single high-flow bolus of contrast material is administered. The images are post-processed using automated or semiautomated perfusion software using deconvolution algorithms to create multiple CTP maps including cerebral blood flow $(\mathrm{CBF})$, cerebral blood volume $(\mathrm{CBV})$, mean transit time acute changes, with low scores predicting less favorable outcome. Software packages are being developed to automate ASPECTS scoring, as shown here (ASPECTS $=7$ ). (RAPID software images modified from PD Dr. med. Carlo Cereda Medico Caposervizio, Neurocentro (EOC) della Svizzera Italiana, Stroke Center, Servizio di Neurologia, Ospedale Civico, Lugano, Switzerland.) (Reprinted with permission, Springer)

(MTT), time to peak (TTP), time to maximum (Tmax), and delay maps. The commonly used parameter for measuring ischemic core volume is $\mathrm{CBF}$ using threshold of $\mathrm{CBF}<30 \%$ as compared to contralateral tissue. The penumbra (hypoperfused area) volume is commonly measured using the threshold of Tmax $>6 \mathrm{~s}$. Mismatch ratios can be generated using core and penumbral volumes. CTP and CTA results are complimentary, facilitating both accurate interpretation and diagnosis (Fig. 3.3).

There has been a recent surge in the use of automated perfusion software, which is critical when triage and treatment decisions need to be made urgently and every minute counts. At the same time, it is important to be aware of the pitfalls of automated CTP processing. The pitfalls include motion artifact, poor signal-to-noise ratio from a suboptimal contrast material bolus, skull base artifacts, inadequate arterial and 


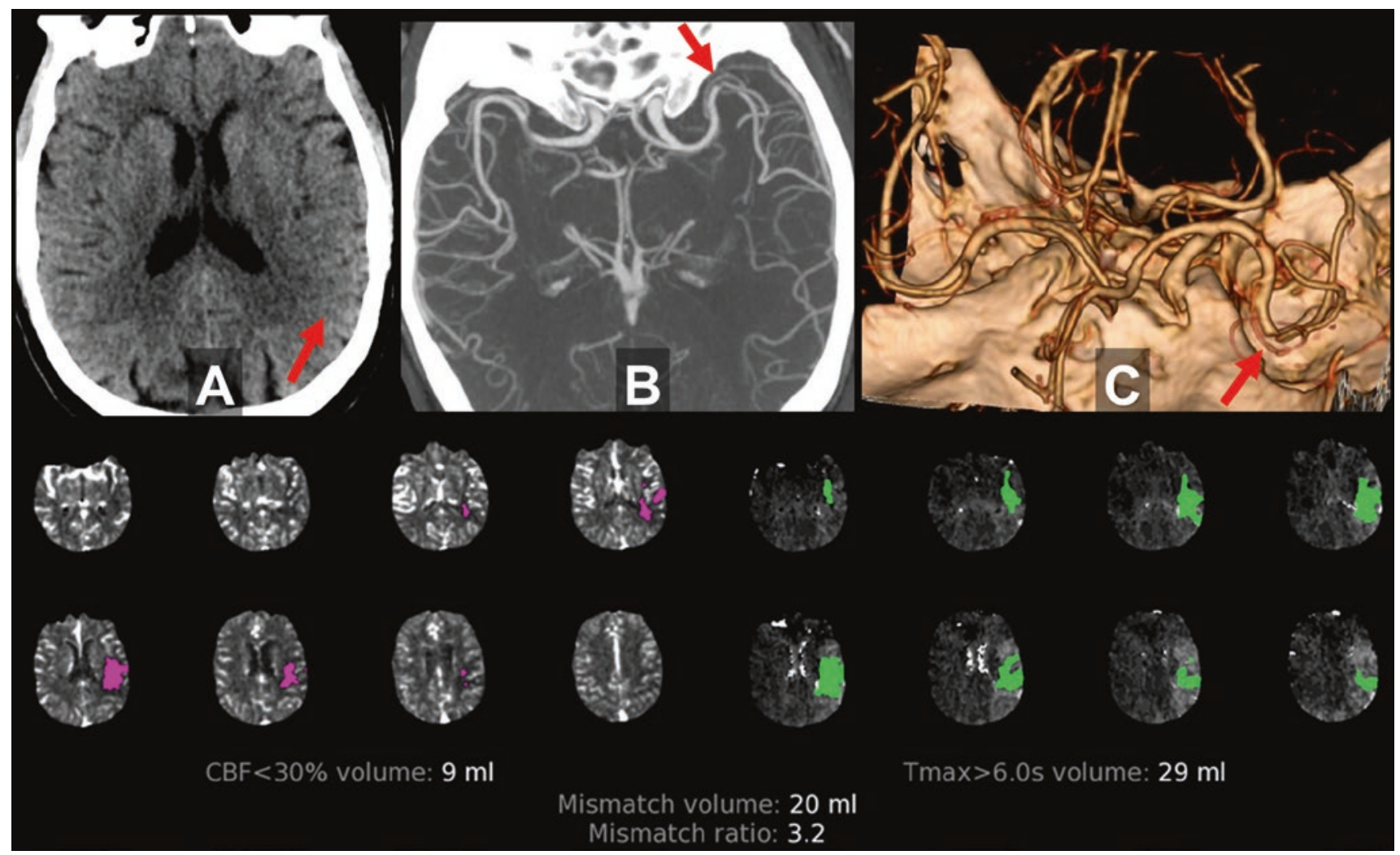

Fig. 3.3 A 74-year-old man 4 days after cardiac bypass surgery with abrupt aphasia and right hemiparesis. (a) non-contrast CT has ASPECTS $=9$, with subtle loss of gray-white distinction in the left angular region (arrow). (b) a subtle filling defect is seen on thick multiplanar reconstructions from the CTA (arrow). CT perfusion (below) shows a large area of ischemia and small mismatch, suggesting a more

venous input selections, and truncated time density attenuation curves. Poor cardiac output and atrial fibrillation can also result in poor contrast bolus. In addition, chronic carotid occlusion can result in false positive penumbra. Furthermore, the thresholds $(\mathrm{CBF}<30 \%)$ may not always capture the true ischemic core, especially for ultra-early acute infarcts and in cases of partial reperfusion. One must be aware of these pitfalls and evaluate the NCCT and CTA in conjunction with CTP for correct interpretation. It is also essential to be cognizant of the fact that there is inherent variation in the different perfusion software packages and post-processing platforms. Even with all of these limitations, CTP is an important tool to assess the ischemic core, penumbra, and mismatch.

\subsection{MRI: Findings, Protocols, and Cases}

An MRI-based approach to stroke imaging offers both advantages and disadvantages compared to CT. Key advantages include maximal sensitivity to ischemia using diffusion MRI, whole brain coverage with dynamic susceptibilitybased perfusion, and lack of ionizing radiation. Disadvantages include availability, need for safety screening, and typically significant stenosis or occlusion is likely present. (c) CTA was postprocessed in 3D to show a proximal M2 occlusion (arrow), missed on initial CTA review due to tortuous overlapping vessels on 2D images. The occlusion site was proven during successful thrombectomy procedure, with good clinical recovery. (Reprinted with permission, Springer)

longer imaging times for similar information. A fully functional MRI triage system requires $24 / 7$ availability near the point of care. Recognizing the urgent nature of stroke triage and to compete with CT, protocols need to be designed to run with a room time of about 10 min or less (Figs. 3.4 and 3.5). Although these requirements can be hard to meet in many centers, MRI should be considered front line in several scenarios (see below Key Points).

\section{Key Points}

Clinical Scenarios Favoring MR over CT

- Wake-up stroke/unknown time of onset

- Late transfer

- Transient ischemic attack

- Posterior fossa/brainstem localization

- Pediatric stroke

- Concern for amyloid or hemosiderosis

- Known or expected complex lesions (e.g., MoyaMoya, stroke vs tumor)

- Iodine allergy 


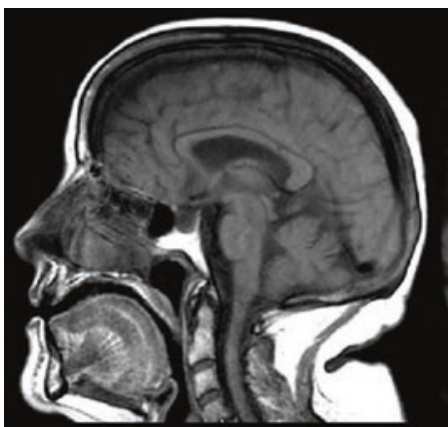

T1

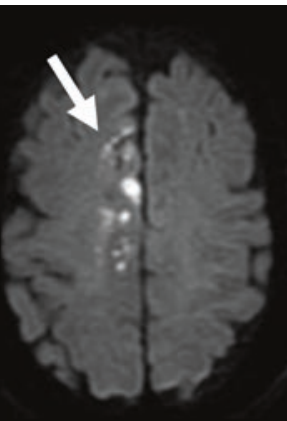

DWI

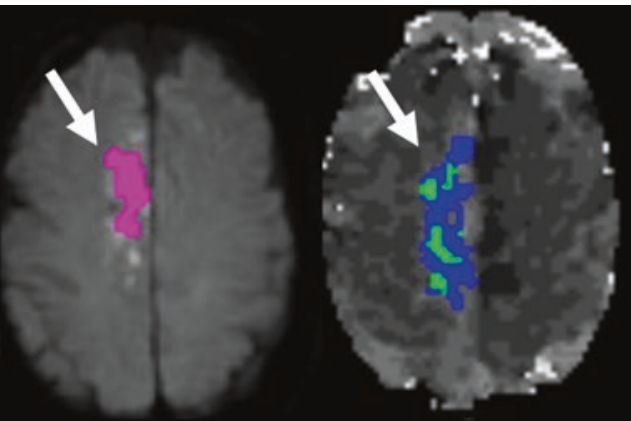

ADC $<620$
Tmax

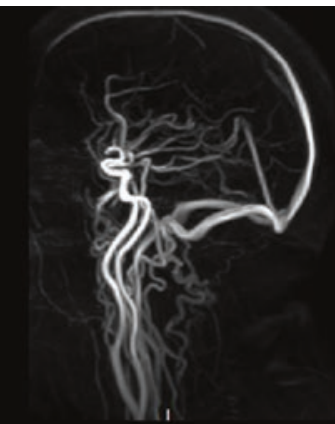

3DPC-MRA

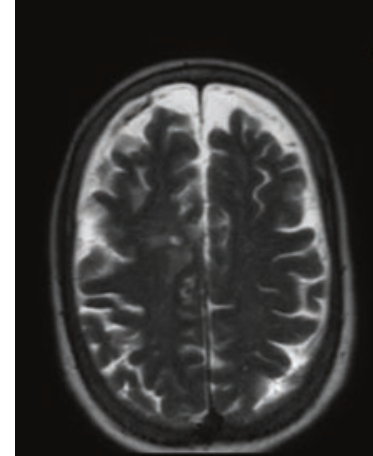

SSFSE-T2

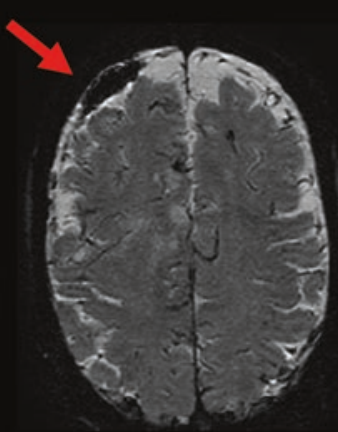

EPI-T2*
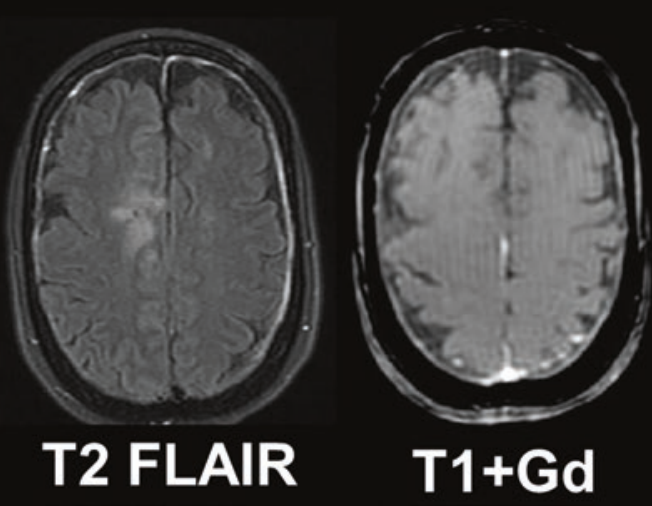

$\mathrm{T} 1+\mathrm{Gd}$

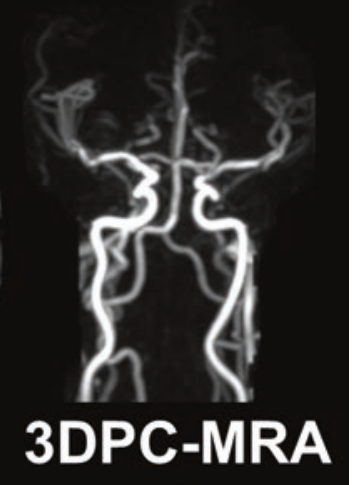

Fig. 3.4 Fast Stroke MRI Screening Protocol. This 64-year-old woman with right anterior cerebral artery stroke 5 days earlier was referred due to falls and worsening leg weakness. The screening protocol shown took $8 \mathrm{~min}$ of total room time, including, in order: sagittal T1 (30 s), DWI (30 s), and dynamic perfusion (75 s). Once completed these were sent to RAPID, with automated mismatch maps constructed (thresholded ADC and Tmax) which returned to PACS in about 3 min, while the post-contrast sequences finished up. Post-contrast images included SSFSE T2 (15 s), EPI-GRE T2* (25 s), 2D FLAIR (70 s), and a 3D phase contrast MRA sequence (130 s) using velocity encoding $50 \mathrm{~cm} / \mathrm{s}$ to capture large arterial or venous occlusions. The MRA sequence was

\subsection{Neurocognitive Approach to Acute Stroke Imaging: Thinking, Fast and Slow}

Whether CT or MRI is used, the time pressure and complexity of large stroke protocols is a challenge for radiologists and neurovascular specialists. In the rush to make key decisions, we run the risk of "having blinders on"-looking for just what we think should be there, and overlooking other stroke territories or mimics. Other members of the stroke team are typically focused on their own key portions of workflow, so it is the radiologist who must help see the big picture and make both an accurate diagnosis and recommendations. A disci- designed with large field of view to capture the carotid bifurcations in the neck, then post-processed to produce both targeted MIP vascular reconstructions from the phase components, and also nominally T1-weighted images in three planes from the magnitude components (only axial shown). There is an early subacute right ACA infarction without large vessel occlusion or significant penumbra (white arrows), as well as acute on chronic hemispheric subdural hematomas, right larger than left (red arrow). Careful construction of fast protocols can refine both stroke diagnosis and capture other significant lesions, including stroke mimics plined search pattern and brief pause at the time of reporting will add value and help achieve these goals. This philosophy is inspired by Daniel Kahneman's book "Thinking, Fast and Slow [17]." In our adaptation of Kahneman's construct, radiologists can harness their innate fast, reflexive thinking patterns to very quickly find and report the key findings of an exam, but they must also impose a slower, orderly approach to drive a less biased, more disciplined exam search pattern and conclusions. Quick action is needed in stroke evaluation but it must always be tempered by a (slightly) slower phase to find unexpected lesions and consider stroke mimics. This essentially boils down to: "Look for what you are supposed to look for-but don't forget about everything else!" 


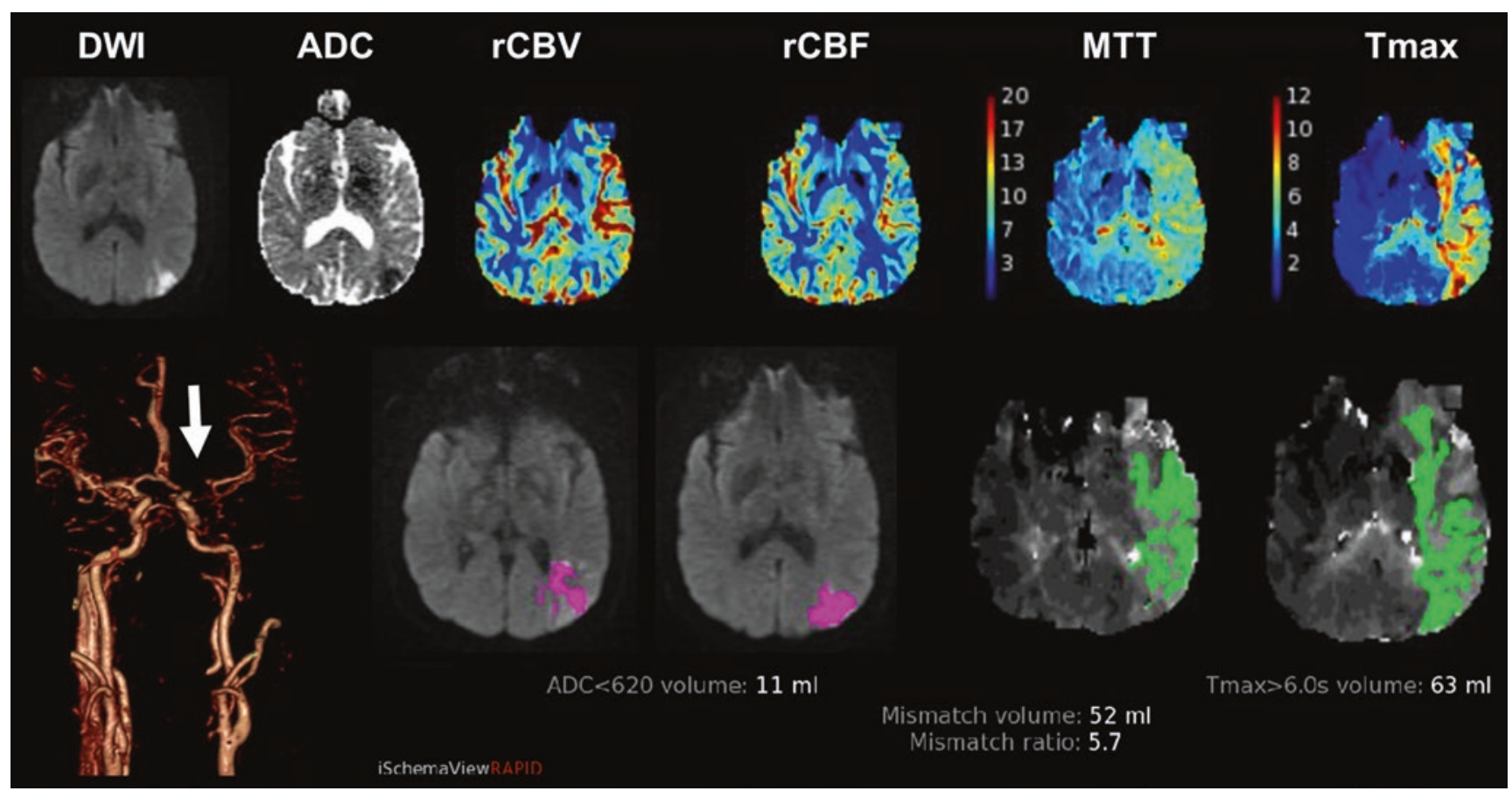

Fig. 3.5 MRI-based triage in a 46-year-old man with global aphasia and right hemiparesis for more than $4.5 \mathrm{~h}$. Upper row shows a small diffusion lesion with low ADC, with much larger perfusion defect by qualitative "eyeball" method. Lower panel shows left carotid terminus near-total occlusion on TRICKS MRA (arrow), with good access via patent cervical carotid. Quantitative thresholded maps made using
RAPID software show a favorable "target mismatch" pattern: a small estimated core infarct (ADC $<620=11 \mathrm{~mL}$, in pink) but large significant perfusion defect (Tmax $>6 \mathrm{~s}=63 \mathrm{~mL}$, in green), for an estimated mismatch volume/penumbra $=52 \mathrm{~mL}$. He underwent successful endovascular therapy and made a complete clinical recovery. (Reprinted with permission, Springer)

\section{Key Points}

Acute Stroke Imaging: Thinking, Fast and Slow

CT/MR exam analysis Search pattern $\rightarrow$ Findings

Fast Hemorrhage Ischemia/infarction Vascular occlusion Perfusion defect

Slow Size/extent/ASPECTS Core and penumbral pattern

Collateral status Non-MCA territories Atherosclerotic disease Second lesions

Stroke mimics

"Corner shot" incidentals Address clinical question Recheck history for clues
Report impression/ conclusion Summary $\rightarrow$ Synthesis Hemorrhage-present or not

Ischemia: location and extent

Vascular occlusion details, site(s) Mismatch and penumbral pattern if any

Differential diagnosis

- What else could it be?

- Pause and consider mimics by category

Mechanistic implications of pattern

Etiology and prevention of recurrent stroke (e.g., non-calcified plaque or

carotid web)

Recommendations

\subsection{Optimizing Imaging Workflow}

Imaging is increasingly integrated into the workup of an acute stroke case and it is critical to establish time efficient imaging protocols. Even though the treatment time windows have been extended for up to $24 \mathrm{~h}$, time remains a crucial variable for good clinical outcomes. A few key elements of an optimal workflow in acute stroke are highlighted below.

1. Ideally, it is important to notify the imaging team as soon as a stroke patient is identified (for example, using a "Code Stroke" pager), which can be during transportation or in the emergency department. Serial group pages or web-based applications serve to update the entire team simultaneously, following the patient's location, streamlining communication, and coordinating care during triage. Patients can be taken straight to CT from ambulance, bypassing the ER evaluation to minimize "door to needle' and "door to groin" times.

2. Imaging including CT angiogram and perfusion studies should not delay the administration of intravenous thrombolysis or EVT. A parallel workflow should be utilized; 
IV tPA can be started in the CT scanner for qualified patients after a non-contrast CT is obtained, as CTA/CTP is being obtained.

3. It is not necessary to delay contrast administration to check renal function. As per the AHA/ASA guidelines, vascular imaging can be obtained without creatinine/GFR check in patients without a history of renal impairment. There is now convincing evidence that in the absence of a history of renal impairment, there is a very low risk of transient, contrast-induced nephropathy.

4. Patient triage decisions are mostly made on the scanner console and hence it is important for the radiologist to be involved in quick interpretation, for example, a NCCT images or DWI sequences should be sent to PACS workstation without waiting for perfusion or angiographic images.

5. Automated perfusion imaging is becoming standard of care and can expedite the workflow. All recent EVT trials used automated software for post-processing of the perfusion studies giving volumetric core, penumbra, and mismatch according to methods used in clinical trials. Processing of the perfusion images on a separate workstation can produce delays along with user variability.

6. The use of automated ASPECTS and automated LVO detection using artificial intelligence (AI) algorithms is increasingly being used by stroke teams, requiring the current and future generation of radiologists to adapt to this new workflow. Multiple expanded uses of AI are in development for use in stroke triage (see below Key Points).

7. It is important for the radiologists to be at the helm for designing efficient workflow at their institution. It is equally important to work in close collaboration with the technologists and stroke team to organize this workflow.

\section{Key Points}

Stroke Imaging of the Future: Opportunities for Artificial Intelligence

- Robust communication systems optimizing regional triage/ambulance workflow and transfer decisions

- Data-driven individualized treatment options

- Radiology reading worklist prioritization based on ordering context, clinical status, and lesion detection algorithms

- Automated radiology exam screening/scoringASPECTS, large vessel occlusion detection, collateral scoring, volumetric core, and penumbral calculations

- Functional deficit risk predictions based on clinical features, lesion location, and volume (at triage and follow-up recovery phase)

\subsection{Stroke Mimics}

Stroke mimics or non-ischemic etiologies represent up to one-third of cases of new neurological deficits [18]. It is important to identify these mimics correctly to avoid unnecessary acute treatment. MRI has a distinct advantage in identifying stroke mimics and narrows the differential as compared to CT-based workup. Multiple etiologies can mimic acute or subacute ischemic stroke including vascular, metabolic, medication-related, infectious, inflammatory, neoplastic, and traumatic etiologies [19]. Common stroke mimics include seizures, migrainous aura, venous thrombosis, PRES, and neoplasms [20].

Seizures: Seizures are one of the most frequent stroke mimics, particularly in patients with presenting with Todd's paresis or postictal aphasia/dysphasia. Seizure related cortical signal abnormalities may have associated DWI abnormality. The distinguishing features from an arterial infarct include a nonvascular distribution, gyral or leptomeningeal enhancement, and absence of vascular occlusion. Signal changes are usually reversible but may progress to cortical laminar necrosis or focal atrophy. There may be mild hyperperfusion or a normal perfusion pattern in the epileptic region.

Migraine: An acute migrainous aura may mimic an acute stroke including motor symptoms (hemiplegic migraine). The neuroimaging is usually normal but uncommonly may show restricted diffusion. The perfusion abnormalities are frequent in these cases, usually with hypoperfusion at onset, sometimes followed by hyperperfusion. An important point to note is that usually more than one vascular territory is affected, in contrast to the hypoperfusion pattern of a single vascular territory in an acute infarct [21].

Neoplasms: Patients with neoplasms may present with sudden attacks of "stroke like" symptoms. This is important to recognize not only to avoid unnecessary thrombolytic treatment but also not to delay recognizing and managing a brain tumor. A common misinterpretation occurs when tumors are small, cortical, in an arterial distribution and have varying enhancement pattern (mimicking a subacute infarct-see Fig. 3.6). Furthermore, DWI can show varying signal characteristics depending on the cellularity of the tumor. Perfusion maybe be helpful especially if there is increased perfusion including increased CBV in a high-grade glioma (as compared to the expected low $\mathrm{CBV}$ in an acute infarct).

Venous infarction: Cerebral venous thrombosis is a lifethreatening neurological condition which should not be missed. A venous infarction has a different topography as compared to arterial infarct; distribution depends on the location of the venous thrombosis but usually lacks an arterial distribution. The DWI abnormality can be variable in a venous infarct. Venous infarcts can also demonstrate a flame shaped hemorrhage. Most of the CTA studies have an adequate venous opacification and evaluation of the 


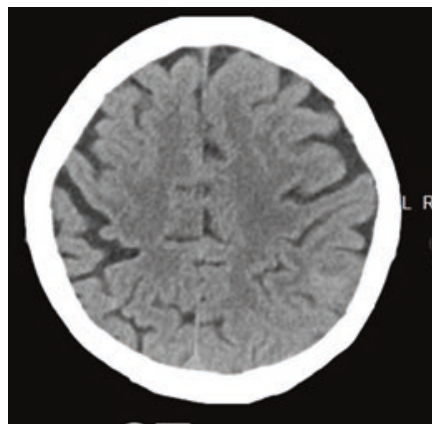

CT -

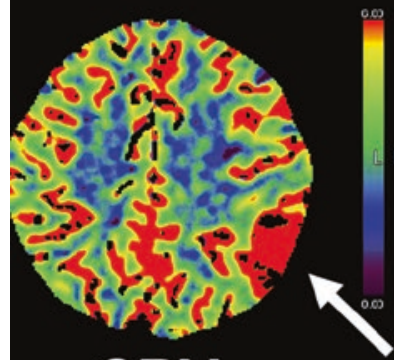

CBV
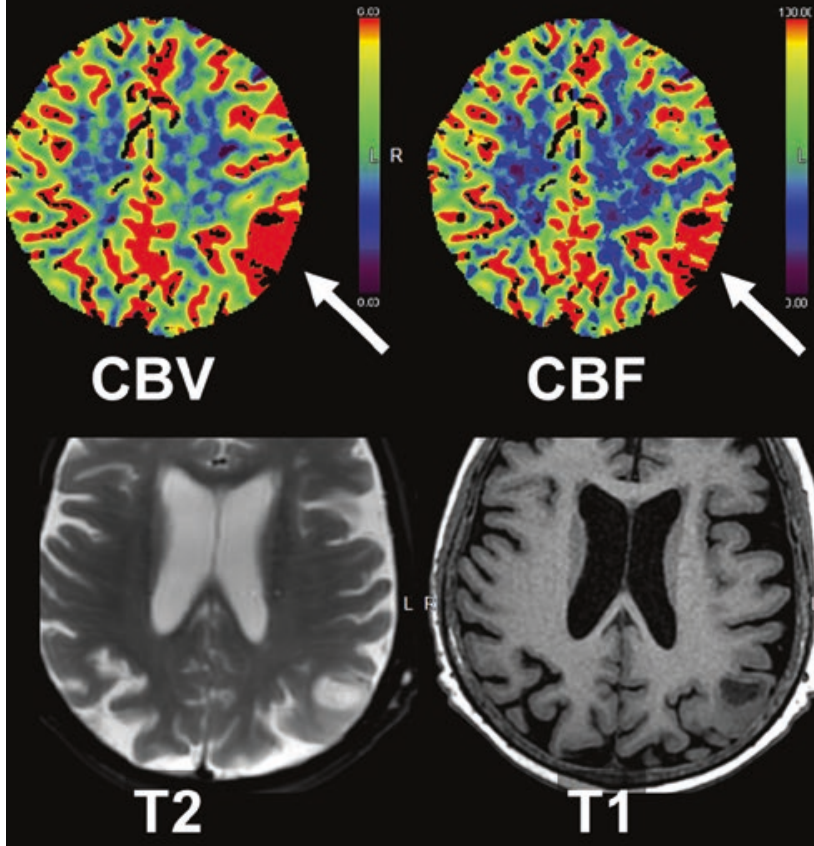

CBF

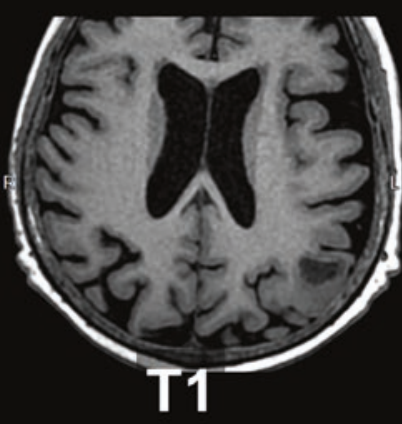

Fig. 3.6 Stroke mimic: tumor. This 83-year-old woman presented to an outside hospital with dysarthria. The emergency stroke CT protocol showed a focal left parietal lesion with curvilinear cortical enhancement and focal elevation of blood volume and blood flow (white arrows), with normal to fast perfusion transit times. This was inter-

venous sinuses should be part of the check list of a CTA interpretation (Fig. 3.7). MR can have variable signal depending on the age of the thrombus. Cortical venous thrombosis can present with focal convexity subarachnoid hemorrhage and is best seen on gradient-echo T2* or susceptibility-weighted sequences.

Posterior reversible encephalopathy syndrome (PRES): PRES is an under recognized entity in the ED with presenting symptoms of headaches, seizures, altered mental status, and visual changes or loss. It is a clinical radiological diagnosis characterized by transient failure of vascular autoregulation leading to multifocal vasogenic edema. Patients with malignant hypertension, eclampsia, on chemotherapy, or taking post-transplant drugs seem particularly susceptible. Typically, the PRES lesions are bilateral, cortical-subcortical, non-enhancing and involve predominantly the parietal-

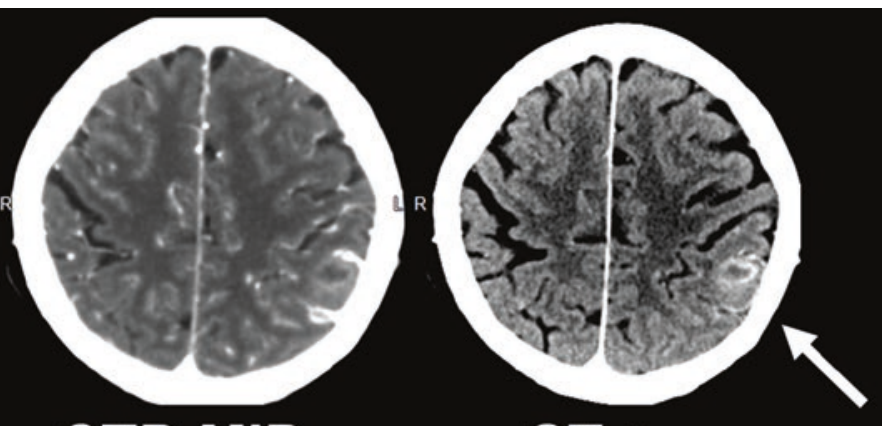

CTP-MIP
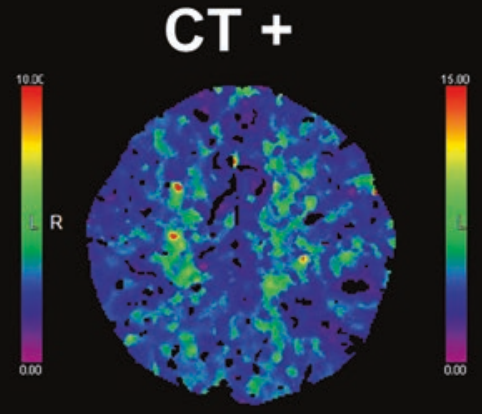

TTD

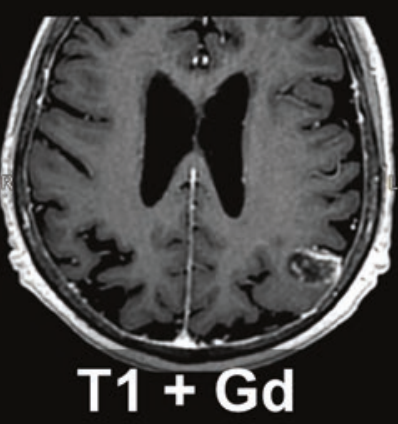

FLAIR + Gd preted as subacute infarction with gyral enhancement and "luxury perfusion." As symptoms progressed, a follow-up MRI 1 month later showed an expansile heterogeneously enhancing lesion, pathologically proven as glioblastoma

occipital regions. Uncommon PRES patterns include posterior fossa involvement, diffusion restriction, enhancement, and hemorrhage (Fig. 3.8).

Subdural hematoma: Patients with subacute to chronic subdural hematoma can present with clinical symptoms masquerading as stroke including confusion, ataxia, and hemiparesis. These are easy to recognize on CT and MRI given their characteristic imaging findings (Fig. 3.9).

Additional entities mimicking acute arterial ischemic stroke include infection (encephalitis, cerebritis, abscess, meningitis, sepsis), toxic-metabolic abnormalities (hypoglycemia, hepatic encephalopathy, medications, and illicit drugs), demyelinating disease, and MELAS (mitochondrial encephalopathy, lactic acidosis, and stroke-like events). Knowledge of these mimics is imperative to minimize incorrect or delayed diagnosis. 


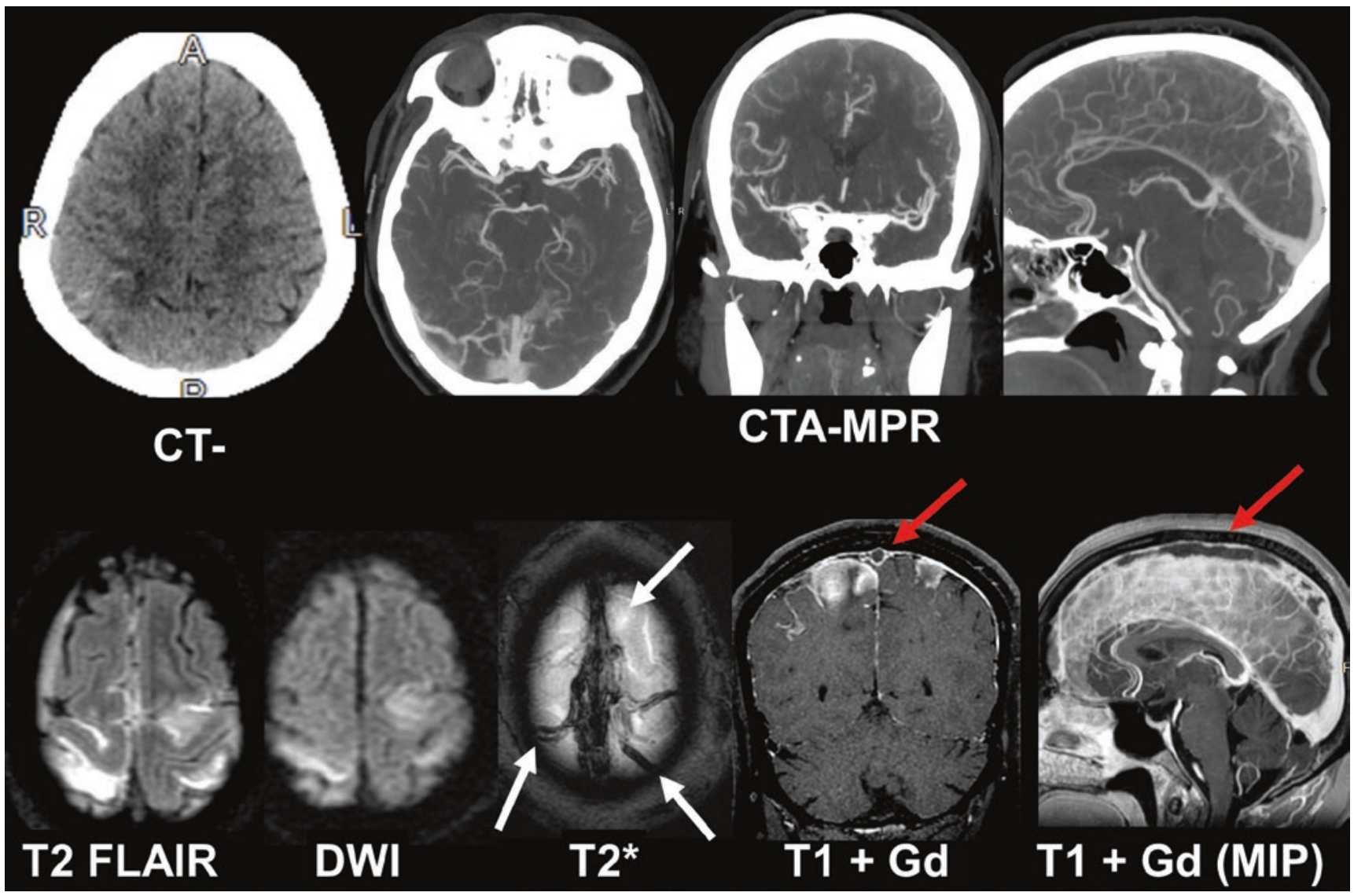

Fig. 3.7 Arterial stroke mimic: venous infarction. This 48-year-old woman with a 2-day history of headache presented with sudden left arm paresthesias. An emergency non-contrast CT shows heterogeneous density and swelling in the right parietal lobe, with linear intrasulcal density suggesting subarachnoid blood. CT perfusion was symmetrical and the CTA was initially interpreted as normal. She worsened and so was referred for MRI $6 \mathrm{~h}$ later (lower panel), showing signs of bilateral hemorrhagic venous infarction, thrombosed superior sagittal sinus, and cortical veins (white arrows), and corresponding dural sinus filling defects on post-contrast images (red arrows). In retrospect, an "empty delta" sign was clearly present in the sagittal sinus on the initial coronal and sagittal CTA reconstructions, but missed due to a focused review done in haste

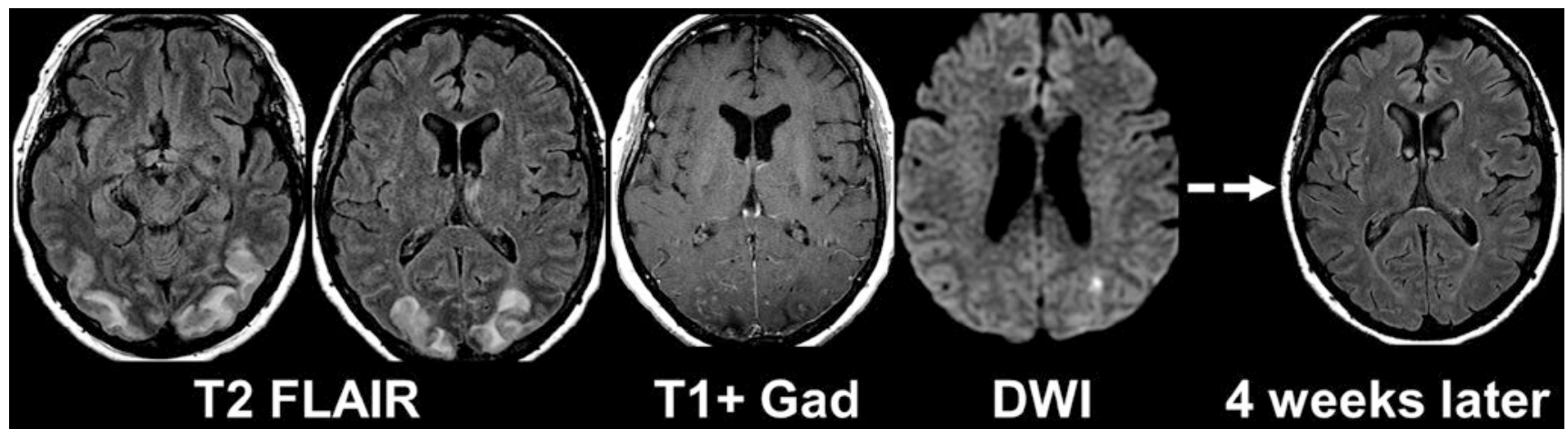

Fig. 3.8 Stroke mimic: PRES. This 55-year-old presented with acute headaches and visual disturbances. MRI demonstrates bilateral, symmetric signal abnormalities consistent with vasogenic edema in the parieto-occipital region, in a classic PRES-like pattern. There is lepto- meningeal congestion but no abnormal parenchymal enhancement, and only a small focus of diffusion restriction. Follow-up FLAIR at 4 weeks shows complete resolution of the signal abnormalities 


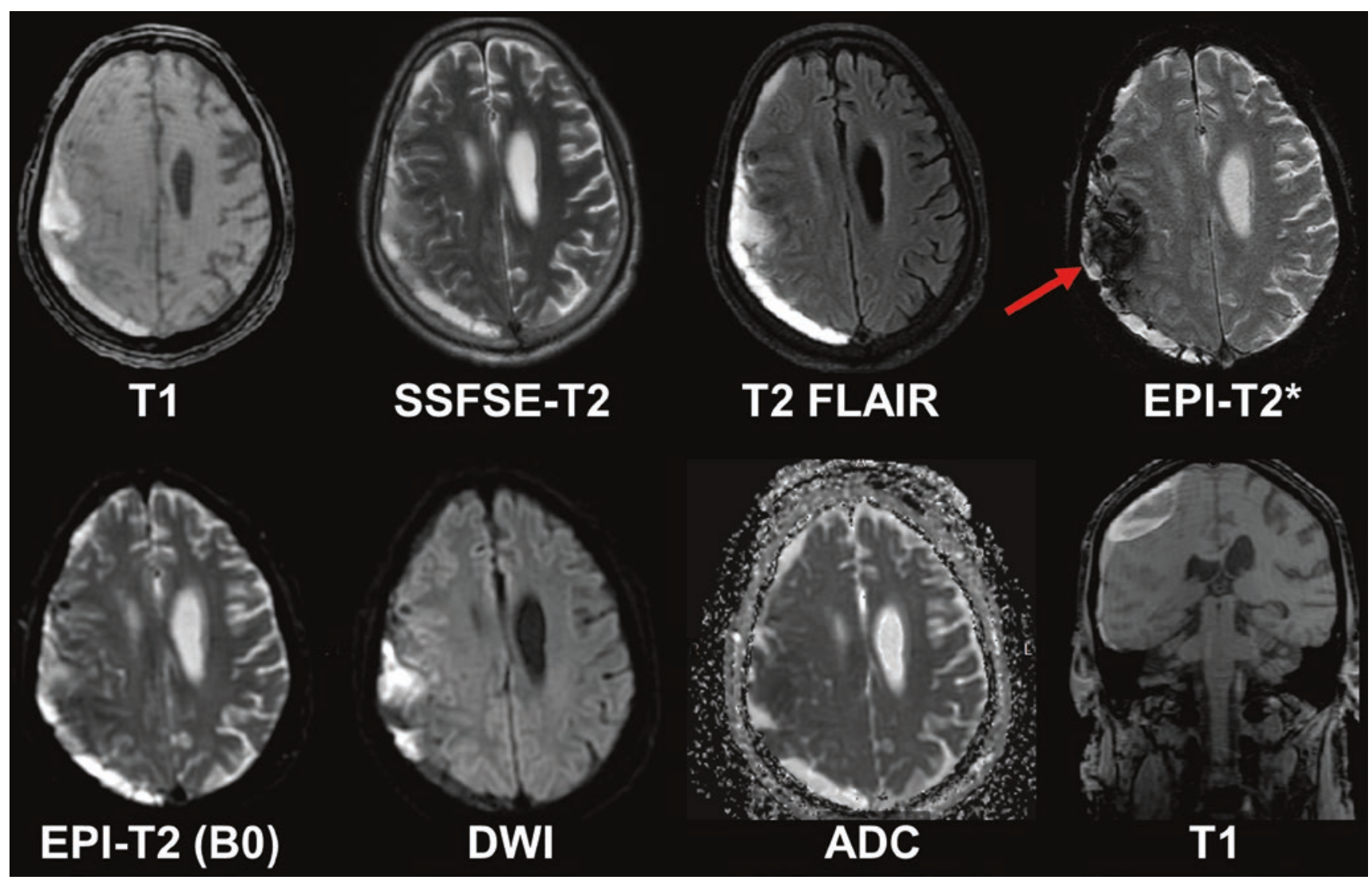

Fig. 3.9 Stroke mimic: subdural hematoma, diagnosed by fast screening MRI protocol. This 58-year-old man presented with abrupt left hemiparesis and confusion. A stroke code was called in the emergency department due to concern for right MCA occlusion. A non-contrast fast screening MRI protocol was obtained in $<7$ min of imaging time

\section{Take-Home Messages}

- Streamline and coordinate stroke triage across your facility

- Build fast yet comprehensive imaging protocols

- Identify extent of acute ischemia, large vessel occlusions, and mismatch/penumbral patterns if any

- Get the right diagnosis - distinguish true strokes from stroke mimics

\subsection{Concluding Remarks}

Rapid stroke imaging triage is challenging but is key to improving outcomes for patients with acute ischemic stroke. Radiologists are at the helm of the changing landscape of (including normal MRA-MRV, not shown). An unexpected right convexity subdural hematoma causes mass effect at the fronto-parietal junction. Predominantly T1 hyperintense signal is consistent with methemoglobin, with focal blooming on $\mathrm{T} 2 *$ (arrow) indicative of an acute intracellular component

acute stroke. Streamlined protocols, workflow, and analysis focus on getting the right diagnosis, adding value not just for acute stroke but also for stroke mimics.

\section{References}

1. Powers WJ, Rabinstein AA, Ackerson T, et al. 2018 guidelines for the early management of patients with acute ischemic stroke: a guideline for healthcare professionals from the American Heart Association/ American Stroke Association. Stroke. 2018;49(3):e46-e110.

2. Turc G, Bhogal P, Fischer U, et al. European stroke organisation (ESO)- European Society for Minimally Invasive Neurological Therapy (ESMINT) guidelines on mechanical thrombectomy in acute ischemic stroke. J Neurointerv Surg. 2019;11(6): $535-8$.

3. Berkhemer OA, Fransen PS, Beumer D, et al. A randomized trial of intraarterial treatment for acute ischemic stroke. N Engl J Med. 2015;372(1):11-20. 
4. Goyal M, Demchuk AM, Menon BK, et al. Randomized assessment of rapid endovascular treatment of ischemic stroke. N Engl J Med. 2015;372(11):1019-30.

5. Jovin TG, Chamorro A, Cobo E, et al. Thrombectomy within 8 hours after symptom onset in ischemic stroke. N Engl J Med. 2015;372(24):2296-306.

6. Saver JL, Goyal M, Bonafe A, et al. Stent-retriever thrombectomy after intravenous t-PA vs. t-PA alone in stroke. N Engl J Med. 2015;372(24):2285-95.

7. Campbell BC, Mitchell PJ, Kleinig TJ, et al. Endovascular therapy for ischemic stroke with perfusion-imaging selection. N Engl J Med. 2015;372(11):1009-18.

8. Nogueira RG, Jadhav AP, Haussen DC, et al. Thrombectomy 6 to 24 hours after stroke with a mismatch between deficit and infarct. N Engl J Med. 2018;378(1):11-21.

9. Albers GW, Marks MP, Kemp S, et al. Thrombectomy for stroke at 6 to 16 hours with selection by perfusion imaging. N Engl J Med. 2018;378(8):708-18.

10. Thomalla G, Simonsen CZ, Boutitie F, et al. MRI-guided thrombolysis for stroke with unknown time of onset. N Engl J Med. 2018;379(7):611-22.

11. Ma H, Campbell BCV, Parsons MW, et al. Thrombolysis guided by perfusion imaging up to 9 hours after onset of stroke. N Engl J Med. 2019;380(19):1795-803.

12. Rowley HA, Vilela P. Brain ischemia: CT and MRI techniques in acute stroke. In: Hodler J, Kubik-Huch RA, von Schulthess GK, editors. Diseases of the brain, head and neck, spine 2016-2019: diagnostic imaging. Cham: Springer International Publishing; 2016. p. 37-47.
13. Rowley HA. The four Ps of acute stroke imaging: parenchyma, pipes, perfusion, and penumbra. AJNR Am J Neuroradiol. 2001;22(4):599-601.

14. Lev MH, Farkas J, Rodriguez VR, et al. CT angiography in the rapid triage of patients with hyperacute stroke to intraarterial thrombolysis: accuracy in the detection of large vessel thrombus. J Comput Assist Tomogr. 2001;25(4):520-8.

15. Miteff F, Levi CR, Bateman GA, Spratt N, McElduff P, Parsons MW. The independent predictive utility of computed tomography angiographic collateral status in acute ischaemic stroke. Brain. 2009;132(Pt 8):2231-8.

16. Menon BK, Smith EE, Modi J, et al. Regional leptomeningeal score on $\mathrm{CT}$ angiography predicts clinical and imaging outcomes in patients with acute anterior circulation occlusions. AJNR Am J Neuroradiol. 2011;32(9):1640-5.

17. Kahneman D. Thinking, fast and slow. New York: Farrar, Straus and Giroux; 2011.

18. Merino JG, Luby M, Benson RT, et al. Predictors of acute stroke mimics in 8187 patients referred to a stroke service. J Stroke Cerebrovasc Dis. 2013;22(8):e397-403.

19. Liu X, Almast J, Ekholm S. Lesions masquerading as acute stroke. J Magn Reson Imaging. 2013;37(1):15-34.

20. Adam G, Ferrier M, Patsoura S, et al. Magnetic resonance imaging of arterial stroke mimics: a pictorial review. Insights Imaging. 2018;9(5):815-31.

21. Floery D, Vosko MR, Fellner FA, et al. Acute-onset migrainous aura mimicking acute stroke: MR perfusion imaging features. AJNR Am J Neuroradiol. 2012;33(8):1546-52.

Open Access This chapter is licensed under the terms of the Creative Commons Attribution 4.0 International License (http://creativecommons. org/licenses/by/4.0/), which permits use, sharing, adaptation, distribution and reproduction in any medium or format, as long as you give appropriate credit to the original author(s) and the source, provide a link to the Creative Commons license and indicate if changes were made.

The images or other third party material in this chapter are included in the chapter's Creative Commons license, unless indicated otherwise in a credit line to the material. If material is not included in the chapter's Creative Commons license and your intended use is not permitted by statutory regulation or exceeds the permitted use, you will need to obtain permission directly from the copyright holder. 